\title{
AfriQue, LANGUES EN DANGER ET DIVERSITÉ LINGUISTIQUE
}

\author{
Sylvie Voisin \\ Aix Marseille Université - DDL UMR 5596
}

\section{Résumé}

Cet article se focalise sur la problématique des langues en danger en Afrique et plus particulièrement nous nous interrogeons sur l'affirmation selon laquelle la situation de ce continent est moins préoccupante que dans d'autres régions du monde. Après un rappel rapide de ce que l'on entend par langues en danger et des différents éléments considérés comme accélérant un phénomène "naturel » pour les langues, nous nous focalisons sur trois facteurs souvent invoqués et qui semblent induire cette vision particulière de la position des langues africaines face à la problématique des langues en danger. Nous nous attacherons à voir si ces facteurs sont suffisants pour expliquer ce constat. Nous tenterons de voir si la sauvegarde de la diversité linguistique passe nécessairement par la description et de la documentation des langues en danger hors de ce continent.

\section{Mots-clés}

Langues en danger, Afrique, diversité linguistique

\begin{abstract}
This article focuses on the issue of endangered languages in Africa and questions the assertion that the language endangerment situation in Africa is less worrying than that in other regions. After a brief discussion of what is meant by endangered languages and the different elements which can be considered to be speeding up the "natural process" of language death, this article will focus on three oft-cited factors that seem to suggest that African languages are in a less precarious situations than other endangered languages. We shall also explore whether the preservation of linguistic diversity on other continents necessarily involves description and documentation of endangered languages.
\end{abstract}

\section{Keywords}

Endangered languages, Africa, Linguistic diversity

L'objectif de cet article n'est pas de dresser un bilan des langues menacées sur le continent africain. Il existe dans la littérature des articles de référence sur la question. Mon propos se focalisera ici sur la position donnée à l'Afrique dans la problématique des langues en danger. L'Afrique est un des continents qui atteste le plus de langues. Un tiers des langues parlées actuellement dans le monde sont 
des langues africaines. Pour autant, lorsqu'il est question de « langues en danger », l'Afrique est généralement considérée comme l'un des continents pour lesquels la situation est parfois présentée comme moins préoccupante qu'ailleurs. Nous nous attacherons ici à comprendre quelles sont les raisons qui poussent certains linguistes à faire ce constat.

Pour cela nous reviendrons sur quelques points récurrents dans les travaux qui semblent expliquer cette vision particulière posée sur les langues africaines. Le premier tient compte de la connaissance des langues et des reconstructions de familles. Le second s'appuie sur la vitalité des langues locales face aux langues officielles. Le troisième repose sur la spécificité du plurilinguisme en Afrique. Nous tenterons de voir si les différences que révèlent ces trois critères sur la question des langues en danger en Afrique aboutissent effectivement au constat d'une situation moins préoccupante. Nous reviendrons sur le fait que certains de ces critères ne sont pas nécessairement adaptés à la situation africaine. Nous reprendrons rapidement les différents facteurs considérés comme accélérant un phénomène « naturel » pour les langues, avec une mise en perspective de ces facteurs sur l'Afrique. Nous aborderons également la question de la diversité souvent invoquée pour expliquer l'urgence de la description des langues menacées. Nous tenterons de voir si la sauvegarde de la diversité linguistique passe en priorité par la description et la documentation des langues en danger hors du continent africain.

\section{LANGues En DANGER (LED) Et Afrique}

Les langues ont toujours disparu parce que les phénomènes liés à leur disparition ont toujours existé et sont liés aux sociétés mêmes. Les facteurs mis en cause sont la disparition des communautés (par catastrophes naturelles, guerres... ${ }^{1}$ ) ou les cas de conquête, colonisation, populations en contact conduisant à une assimilation culturelle et linguistique.

Nichols (1999, p. 274-275), dans sa présentation sur l'expansion des langues à travers le monde depuis l'origine du langage, fait coïncider la première période de perte de diversité linguistique avec la fin de l'ère glaciaire (troisième et dernière grande étape de déplacement et de peuplement de la terre par Homo Sapiens).

Plus près de nous, certaines populations de l'Afrique de l'Est et du Sud ont été absorbées ou repoussées par les différentes vagues d'expansion des Bantous dont

1 Ferro $(1994 ; 2003)$ estime que 4,1 millions de subsahariens furent déportés du « fait des Arabes » contre 13,2 millions du « fait des Européens ». Entre 1880 et 1920, les épidémies et les travaux forcés ont décimé un tiers de la population du territoire, bien plus que l'ensemble des traites pendant des siècles. Si M'Bokolo (1995, p. 165) émet beaucoup de réserve sur la fiabilité de tels chiffres pour ces différentes périodes, il note pour autant qu' " aucun continent n'a connu, sur un temps aussi long $\left(\mathrm{VII}^{\mathrm{e}}-\mathrm{XIX}^{\mathrm{e}} \mathrm{s}\right.$.), une saignée aussi continue et aussi systématique que le continent africain ». 
la première remonterait à 3000 avant notre ère. Cette expansion a eu un impact sur l'ensemble des phylums du continent ${ }^{2}$, y compris sur des populations issues des premières vagues d'expansion bantoue, ce qui rend la classification interne de certaines branches problématique dans plusieurs zones.

La connaissance de l'histoire de ces différents phénomènes en Afrique est pour autant parcellaire. Bien souvent, la tradition orale, source essentielle de cette partie de l'histoire africaine, est complétée et appuyée, entre autres, par l'analyse linguistique $^{3}$. Mais les régions sur lesquelles de telles études sont menées sont focalisées sur les grands groupes linguistiques, sur les zones où les données linguistiques sont conséquentes, ce qui laisse une large part à peine ébauchée voire ignorée.

La problématique des LED telle qu'elle est théorisée aujourd'hui met en avant d'autres facteurs favorisant la disparition des langues, souvent présentés comme des facteurs accélérant un processus « naturel ». Ces facteurs sont liés à la mondialisation et à plus petite échelle à des phénomènes de globalisation et d'urbanisation. Sur cette base, et suite aux ouvrages de Krauss (1992) et de Crystal (2000), les travaux sur les LED se sont multipliés. Les présentations générales sur les LED s'accordent sur le fait que la diversité linguistique est menacée et qu'il est urgent de décrire ces langues avant leur disparition. On trouve dans les travaux sur la question une tendance générale à considérer la situation en Afrique comme différente des zones les plus menacées, ce qui peut conduire à supposer que la situation des langues africaines est moins préoccupante que dans d'autres parties du monde. Ce contraste entre l'Afrique et les autres continents est mis en avant y compris par les spécialistes des langues africaines, même si ces derniers ne vont pas systématiquement jusqu'à considérer la situation comme étant moins préoccupante.

[...] African situation is different from that in the Americas and Australia, where endangered languages are associated with (indigenous) peoples who belong to the "lower" classes of society. This is not generally the case in Africa. (Mous 2003, p. 157)

2 Un cas bien documenté des conséquences de cette expansion est celui de la région des grands lacs. Dans l'aire dite de la vallée du Rift, les différentes vagues de peuplement et de domination ont laissé des traces sur les langues actuelles (Kießling, Mous, et Nurse 2008). On retrouve dans cette zone des cas de simple influence (emprunt, innovation partagée...) et des cas d'assimilation. Les Dahalo sont ainsi considérés, dans certains travaux, comme étant à l'origine des locuteurs d'une langue khoisan parlant aujourd'hui une langue couchitique (Ehret 2000, p. 276).

3 Bien évidemment, on pense aussi à l'archéologie pour compléter cette reconstruction. Cependant, les conditions climatiques, les sols fortement acides dans certaines régions ne sont pas propices à la conservation des vestiges qui résistent mieux dans les pays secs et froids. Il faut ajouter un certain désintérêt, ou un plus fort attrait pour la paléoarchéologie que pour des recherches sur une archéologie moins ancienne dans ces régions. Là encore, lorsqu'elles existent, de telles études sont menées sur de grands groupes. Des études archéologiques sont ainsi venues renforcer les scénarios reconstruits par la linguistique et les traditions orales sur l'expansion des Bantou. 
On the whole, language death appears to be less dramatic on the African continent than in other parts of the world. (Dimmendaal et Voeltz 2007, p. 598)

West African languages are generally holding their own in the face of globalisation and the homogenising forces of the twenty-first century. (Blench 2007, p. 156)

Dans les sections suivantes, nous allons détailler les arguments mis en avant pour parvenir à ce constat, nous tâcherons de voir sur quels faits reposent ces affirmations et si ces différences permettent effectivement de considérer que la sauvegarde de la diversité linguistique ne passe pas nécessairement par la description et la prise en compte des LED en Afrique.

\section{Pourquoi la situation en Afrique est-elle MOINS PRÉOCCUPANTE QU'AILLEURS ?}

Trois caractéristiques spécifiques à l'Afrique sont régulièrement mises en avant pour considérer la situation de l'Afrique comme moins préoccupante.

Il y a tout d'abord le fait que les langues africaines, bien que nombreuses, sont reliées entre elles en seulement 4 phylums et que sur l'ensemble du continent on ne relève que très peu d'isolats.

One of the notable differences between Africa and most other linguistic areas is its relative uniformity. With few exceptions, all of Africa's languages have been gathered into four major phyla, and most recent progress in classification has been in resolving details. The number of undisputed language isolates is very small. By contrast, Australia, Papua and the New World are usually considered diverse at the phylic level and all have numerous isolates or very small phyla. (Blench 2013, p. 43)

While this region [Central America] has a much smaller number of languages than, say, Africa, it has a considerable number of unique languages not related to any others. (Nettle \& Romaine 2000, p. 8)

Il y a ensuite le fait que les langues africaines attestent d'une certaine vitalité face aux langues officielles, langues non autochtones bien souvent indo-européennes.

[...] A number of African languages are now in a stronger socio-political situation than they were twenty years ago and these languages have gained ground against the European official languages: Wolof in Senegal, Bambara in Mali, Setswana in Botswana to name a few. (Mous 2003, p. 157)

While language dynamics in Africa undoubtedly vary as much as elsewhere in the world, overall the recent history of language vitality on this continent questions some of the claims of the rapidly vanishing linguistic diversity that are based on the experience of especially North America and Australia. It is debatable whether the colonial European languages that now function as official languages in Africa are (general) threats to the continent's indigenous vernaculars. (Vigouroux \& Mufwene 2008, p. 2) 
Enfin, les langues minoritaires résisteraient mieux que dans les autres régions du monde du fait de l'existence d'un multilinguisme très ancré dans ces populations.

The African continent has been described as linguistically 'distinct' (Grenoble \& Whaley, 1998, p. 42) because of its highly complex language situation. Apart from the multitude and high concentration of languages, the patterns of language choice and use are remarkably intricate, as most people are multilingual - that is, they speak several languages - and select the language or variety of language they use according to the context. (Batibo 2005, p. 1)

\subsection{Le point sur la relative homogénéité dans les classifications des langues africaines}

Depuis les travaux de Greenberg (1963) (figure 1), les langues africaines sont réparties à l'intérieur des quatre phylums, désignés aujourd'hui sous les termes d'Afroasiatique, Niger-Congo, Nilo-sahara et Khoisan. Dans les travaux de Greenberg, on relevait peu d'isolats.

Si ces classifications ne sont pas remises en cause dans leur ensemble, l'avancée des travaux de description des langues d'Afrique conduit cependant à leur révision partielle. L’apparente homogénéité de ces langues est reconsidérée et plusieurs éclatements sont désormais bien acceptés par la communauté des africanistes. Ainsi, la nouvelle présentation des affiliations des langues africaines de Dimmendaal (2008) (figure 2) est plus conforme aux connaissances actuelles sur les langues africaines que les classifications basées sur les reconstructions de Greenberg traditionnellement présentées.

Dans cette nouvelle présentation des affiliations entre les langues africaines (figure 2), on retrouve 3 des 4 phylums de Greenberg. De nombreuses réorganisations internes de ces phylums sont également visibles. Elles sont assez souvent le résultat de la sortie d'un groupe de langues qui constitue désormais un isolat, comme par exemple les langues oubanguiennes ou les langues mandé. Autre cause de cette refonte des classifications, la sortie d'une branche pour former une famille distincte, comme par exemple la famille dite ouest-atlantique divisée aujourd'hui en deux familles toujours rattachées au Niger-Congo, mais ne présentant pas de liens plus étroits qui pourraient les maintenir sous un même regroupement. On distingue ainsi aujourd'hui la famille des langues mel et la famille des langues atlantiques. Le phylum khoisan est maintenant présenté en 3 familles distinctes dont les langues partagent certes des caractéristiques communes, mais ne peuvent pour autant être considérées comme apparentées.

Cette nouvelle configuration montre que les avancées opérées sur des zones géographiques particulières ou des familles de langues jusqu'alors peu décrites ont conduit à des réorganisations assez conséquentes des premières classifications. Les enquêtes centrées sur des pays ou zones particulières, sur une famille ou groupe 
dans son ensemble mettent souvent en évidence des apparentements plus distants, des isolats et redessinent la carte des affiliations.

\begin{tabular}{|l|l|} 
Afroasiatique & Niger-Congo \\
\hline Sémitique & Kordofanien (5gpes) \\
\hline Couchitique & Mandé \\
\hline Omotique & Ouest-atlantique \\
\hline Berbère & Kwa \\
\hline Tchadique & Gur \\
\hline Egyptien & Adamawa \\
\hline & Oubanguien \\
\hline Nilo-sahara & Dogon \\
\hline Songhaï & Ijoïd \\
\hline Saharien & \\
\hline Maban & Khoisan \\
\hline Fur & Nord khoisan \\
\hline Soudanique central & Khoisan central \\
\hline Soudanique oriental & Sud khoisan \\
\hline Koman & sandawe (isolat) \\
\hline Kadu & hadza (isolat) \\
\hline & quelques isolats \\
\hline
\end{tabular}

Figure 1 - Classifications des langues africaines basées sur Greenberg (1963),

schéma réalisé à partir de Heine et Nurse (2000)

\begin{tabular}{|c|l|} 
Afroasiatique & Nord khoisan \\
\hline (réorganisation interne à la marge) & Khoisan central + sandawe, kwadi \\
\hline Nilo-sahara & Sud khoisan \\
\hline (réduit + réorganisation interne) & Koman + gumuz \\
\hline Saharien & Songhaï (groupe dialectal, isolat) \\
\hline Maban & Kadu (groupe, isolat) \\
\hline Fur & Mandé (groupe, isolat) \\
\hline Soudanique central & Oubanguien (groupe, isolat) \\
\hline Soudanique oriental & hazda (langue, isolat - Tanzanie) \\
\hline & laal (langue, isolat - Tchad) \\
\hline Niger-Congo & bangime (langue, isolat - Mali) \\
\hline (réduit + réorganisation interne importante) & dompo (langue, isolat - Ghana) \\
\hline Kordofanien (réduit à 4 gpes) & mpra (langue, isolat - Ghana) \\
\hline Langues Mal & jalaa (langue, isolat - Nigéria) \\
\hline Atlantique & ongota (langue, isolat -Ethiopie) \\
\hline Kwa & shabo (langue, isolat - Ethiopie) \\
\hline Gur & \\
\hline Adamawa & \\
\hline Dogon & \\
\hline Ijoïd &
\end{tabular}

Figure 2 - La classification des langues africaines basée sur la révision proposée par Dimmendaal (2008) 
La description des langues africaines est une tâche immense à peine entamée comparativement à d'autres régions du monde. Certains groupes peuvent assurément être considérés comme bien connus aujourd'hui, comme les langues mandé ou les langues oubanguiennes. Des efforts considérables sont entrepris sur d'autres ensembles (atlantique et khoisan). Cependant, nombre de familles, branches ou groupes restent encore très peu connus. Bien souvent les connaissances sur un groupe reposent sur la description ou l'esquisse d'une seule langue et de quelques informations éparses sur les autres langues qui composent l'ensemble ${ }^{4}$. On peut donc raisonnablement supposer que l'éclatement en cours dans les affiliations des langues africaines ne pourra qu'augmenter avec l'avancée des travaux de descriptions. Cette connaissance partielle des langues doit également être prise en compte dans les recensements faits des LED par continent et par pays que l'on peut supposer amoindris du fait d'une mauvaise connaissance des LED sur ce continent.

\subsection{Grande vitalité des langues africaines face aux langues officielles}

La plupart des langues véhiculaires du continent africain sont des langues autochtones. Les langues officielles, dans la grande majorité des langues indoeuropéennes, ont rarement pris le dessus sur les langues locales.

En Afrique, les langues officielles européennes sont en concurrence directe avec des pidgins, des créoles locaux, des variétés locales des langues officielles, jamais avec des langues véhiculaires africaines. C'est le cas, par exemple, en Côte d'Ivoire entre le nouchi (variété urbaine du français ivoirien) et le français, entre le créole de Guinée Bissau et le portugais en Guinée Bissau, l'anglais libérien et l'anglais américain au Libéria.

Hormis les très rares pays où les langues officielles européennes ont un rôle de langue véhiculaire en dehors des grands centres urbains, comme au Gabon ${ }^{5}$ ou en Angola, les langues officielles ont le plus souvent un rôle de langue de communication inter-communautaire uniquement dans les capitales et pour seulement une partie de la population et/ou un type d'interaction bien précis. Cette particularité des langues officielles européennes distingue en effet l'Afrique d'autres continents ayant connu la colonisation.

L'explication de cette situation repose au moins sur trois points. Tout d'abord, l'existence de langues véhiculaires locales est attestée bien avant la colonisation.

4 Ceci s'explique par le fait que dès le début et jusqu'à récemment, les études sur les langues africaines ont été conduites dans une perspective comparatiste reposant pour l'essentiel sur des listes de mots et de morphèmes. En conséquence, si la connaissance de ce type de données couvre de très nombreuses langues africaines, ce sont aussi les seuls éléments dont on dispose pour beaucoup d'entre elles.

5 Le français est souvent considéré comme une langue à la fois officielle et véhiculaire au Gabon, même si elle a été en concurrence dans cette dernière fonction avec le « sabir » (dit aussi « français militaire ») ou avec le fang selon les régions. 
Le continent africain a toujours connu des contacts de langues intenses, mais la domination d'un groupe pour des raisons politiques ou militaires sur ce continent a plus souvent conduit à des situations de multilinguisme qu'à l'assimilation ${ }^{6}$. Les langues véhiculaires locales sont pour la plupart des langues anciennement liées au commerce (dioula, swahili) ou des langues que les états-vassaux de grands royaumes avaient adoptées dans certaines de leurs pratiques culturelles et/ou sociales. Certaines langues du fait de conquêtes se sont répandues dans des zones plus ou moins importantes. Ces langues véhiculaires, même si elles ne recouvrent pas toujours les pays-nations dessinés à la suite de la colonisation et de la décolonisation, ont pu poursuivre leur rôle de langues de communication entre différentes communautés pendant et après la colonisation (mandingue, wolof, zoulou...). Ensuite, le système de colonisation d'exploitation (Mufwene 2003) explique en partie l'absence de domination des langues officielles sur les langues locales. L'absence d'une colonisation d'habitation n'a pas imposée la langue d'une nouvelle population dominante. Dans la plupart des pays d'Afrique, les colons sont passés par les populations locales dominantes à leur arrivée, renforçant indirectement l'attrait de la langue de ces derniers. L'expansion plus rare de pidgins, créoles ou variétés locales de langues européennes s'explique par l'histoire différente de certaines régions et des découpages imposés par les nouvelles frontières des nations issues de la colonisation. On peut citer par exemple, le cas de l'Afrique du Sud qui a connu deux types de colonisation (exploitation et habitation) aboutissant à l'émergence de l'afrikaans.

Cette faible expansion des langues officielles européennes conduit à présenter les langues africaines comme attestant une certaine vitalité face aux langues officielles.

Ci-dessous, trois éléments sont présentés qui viennent amoindrir cette apparente vitalité des langues africaines. Tout d'abord, la situation générale de l'Afrique ne doit pas masquer le fait que cette situation ne reflète en rien la réalité de certains pays, comme le Gabon ou l'Angola déjà cité. Ensuite, pour les pays africains où la langue véhiculaire est une langue locale, la vitalité des langues africaines ne concerne en fait qu'une partie des langues en concurrence. La véhicularisation de langues africaines dans les centres urbains, mais également dans des régions entières de ces pays, montre effectivement que certaines langues africaines ont pris le pas sur les langues officielles. Ainsi, à la différence d'autres régions du monde,

6 Ces contacts de langues, même s'ils n'ont pas abouti à une assimilation, ont bien évidemment laissé des traces plus ou moins fortes sur les langues en question. Ce sont généralement ces transferts d'une langue à l'autre qui expliquent également les difficultés de classements internes. Certaines langues africaines sont d'ailleurs assez connues pour être « inclassables » ou du moins difficilement classables du fait de ces échanges donnant lieu à des convergences multiples et complexes, comme le songhay ou le sango, pour ne citer qu'elles. 
les langues locales ne disparaissent pas au profit d'une langue officielle venue d'ailleurs. C'est cet aspect qui est pris en compte lorsque l'on parle de vitalité des langues africaines. Cependant ce constat occulte une autre facette de cette même situation, qui est que, si des langues africaines voient leur utilisation s'élargir au-delà de leur propre communauté, cela implique nécessairement que d'autres langues africaines voient leur champ d'action se réduire et sont donc par conséquent menacées. Autrement dit, ce n'est parce que se sont véhicularisés le wolof au Sénégal, le bambara au Mali, le mooré au Burkina-Faso, le lingala en République Démocratique du Congo et le swahili dans plusieurs pays d'Afrique de l'Est, pour ne citer que quelques cas, que l'expansion d'une langue africaine ne s'opère pas non plus sur un grand nombre d'autres langues locales. Même si les conséquences de l'expansion d'une langue africaine sur d'autres langues africaines sont prises en compte, l'argument de la diversité linguistique est souvent invoqué pour indiquer que cette situation place l'Afrique dans une position différente et moins préoccupante que d'autres zones. Le raisonnement de cette position postule que l'impact de l'expansion d'une langue africaine sur une autre langue africaine est moins dommageable pour la diversité linguistique que l'expansion d'une langue « importée » au détriment d'une langue locale, sous entendue génétiquement et par conséquent typologiquement différente. Cet argument est à rejeter doublement puisque d'une part, il n'est pas prouvé, ni prouvable que les langues africaines qui se véhicularisent ne remplaceront à terme que des langues qui leur sont strictement apparentées. D'autre part, les langues africaines si elles peuvent être assemblées dans un nombre restreint de phylums ne sont pas pour autant toutes des langues apparentées et typologiquement similaires. Enfin, même s'il est vrai que pendant longtemps, l'impact des langues des colonisateurs n'a pas eu le même effet sur les langues africaines que sur les langues d'autres régions du monde, comme les Amériques ou l'Australie, cela ne veut pas pour autant dire que cette situation est immuable. Des changements récents semblent venir bouleverser cette configuration. Dans les sections 3 et 4 nous reviendrons respectivement sur la notion de diversité en linguistique, ainsi que sur ces questions de vitalité et de particularité africaine.

\subsection{Multilinguisme et plurilinguisme en Afrique}

Le dernier facteur évoqué, souvent en lien avec le précédent, pour expliquer que la situation est moins préoccupante en Afrique est le multilinguisme qui a cours sur ce continent.

Comme la section précédente le suggère, les situations de multilinguisme ne se résument pas en Afrique à une situation de diglossie ou de triglossie (Batibo 
2005, p. 17). La situation est plus complexe et ne relève pas du champ de la di-/pluriglossie du moins d'une présentation pyramidale et hiérarchisée des langues tant dans la société que dans le répertoire linguistique du locuteur. Toutes les couches de la population d'Afrique sub-saharienne, à de rares exceptions près, ont toujours été et sont encore plurilingues y compris les classes les plus aisées. Le répertoire linguistique des populations africaines comprend assez souvent plus que la langue officielle, la langue maternelle et la langue véhiculaire. Et la hiérarchisation qui est corrélée à cette représentation s'adapte mal à la situation de l'Afrique, i.e. une langue officielle, variété haute, mal maitrisée ou maitrisée de façon inégale face à des langues locales, la langue véhiculaire et la langue maternelle, en position basse, qui seraient elles bien maitrisées.

Le terme de répertoire linguistique renvoie à l'ensemble des langues pratiquées par le locuteur. Les différentes langues en jeu dans le répertoire linguistique sont utilisées pour des situations de communication spécifiques ou adaptées en fonction de l'interlocuteur. L'utilisation d'une des langues du répertoire n'est pas nécessairement le reflet d'une identité assumée et revendiquée par la pratique. Elle est juste adaptée à la situation de communication et des interlocuteurs de l'acte de communication.

Il est fréquent qu'une langue, i.e. une partie du répertoire, soit remplacée par une autre, voire dans bien des cas simplement ajoutée au répertoire linguistique existant. Chaque langue du répertoire reste utilisée dans des situations distinctes et certaines d'entre elles subsistent, mais souvent dans des situations d'utilisation très réduites. La problématique de la disparition des langues en Afrique doit donc être abordée différemment. En effet, si certains contextes appellent la pratique de certaines langues, est-ce que cela signifie pour autant que l'utilisation d'une langue restreinte à un seul domaine suffit à considérer que cette langue n'a pas disparu ? Une « langue » utilisée pour des communications très spécifiques présente-t-elle un degré de vitalité satisfaisant?

En résumé, la tendance de présentation synthétique des filiations des langues africaines est en train d'être révisée avec l'avancée des connaissances. Sur ce point, la diversité linguistique des langues africaines semble être plus importante que ne le laissaient supposer les classifications héritées de Greenberg. Des langues véhiculaires locales ont pris, dans beaucoup de pays du continent, « la place» ordinairement dévolue à la langue officielle, ce qui confirme la résistance de certaines langues africaines. Cette situation se distingue de celles observées dans d'autres régions du monde. Il ne faut pas néanmoins oublier que seule une infime part des langues africaines entre dans cette résistance. Ce dernier point est certes amoindri par l'existence de répertoires linguistiques en Afrique sub-saharienne, mais cela doit nous conduire à considérer la problématique de la disparition des langues 
sous un autre angle. Il est également nécessaire de distinguer le plurilinguisme du monde rural de celui des villes qui semble considérablement changer depuis quelques temps dans certaines régions d'Afrique sub-saharienne.

La situation en Afrique est donc indéniablement différente de celle rencontrée dans d'autres parties du monde. Il nous reste maintenant à voir si cette différence conduit effectivement au constat d'une situation moins préoccupante. Dans les sections suivantes, nous revenons tout d'abord sur la question de la diversité linguistique. Puis, nous présenterons deux points qui doivent être mieux considérés dans l'observation des LED en Afrique.

\section{QU'EST-CE QUE LA DIVERSITÉ EN LINGUISTIQUE ?}

Dans cette section, nous revenons sur le terme de diversité afin de voir si la supposée uniformité des langues africaines échappe à la diversité linguistique que l'on tente de sauvegarder en documentant prioritairement d'autres LED. On peut distinguer trois types de diversité en linguistique.

La diversité linguistique peut renvoyer au nombre de langues parlées auquel on peut ajouter la densité linguistique. L'index de diversité défini par Greenberg (1956) est basé sur le ratio entre nombre de langues et population pour un pays. L'index de diversité de l'Afrique (entre 0.7 et plus de 0.9 selon les pays, à l'exception de quelques pays du Sud de l'Afrique) ${ }^{7}$ peut être rapprochée des autres zones attestant également un fort taux de diversité linguistique comme l'Asie ou la zone du Pacifique. Sur ce point, avec un tiers des langues parlées dans le monde et une densité linguistique très élevée, on peut difficilement dire que l'Afrique sub-saharienne ne présente pas de diversité linguistique.

La diversité linguistique peut également être comprise en nombre d'apparentements distincts (i.e. au-delà desquels on ne peut assembler les langues), ce que l'on nomme la diversité génétique. C'est généralement sur ce critère que l'Afrique est distinguée des autres régions pour lesquelles la diversité génétique est considérée comme plus importante. Si l'on compare le nombre de regroupements, on trouve effectivement beaucoup plus d'apparentements différents aux Amériques qu'en Afrique. Pour la seule Amérique du Sud, Kaufmann (1990 ; 1994) recense 118 familles différentes pour environ 400 langues. Sur ces 118 familles, 70 sont des isolats pour lesquels une langue équivaut à une famille. Ce recensement est assez éloigné des 19 regroupements et des isolats de la dernière classification proposée par Dimmendaal pour l'Afrique (cf. 2.1). Cependant, cette comparaison ne

7 Pour plus de détails sur le ratio pays par pays et une représentation graphique de cette diversité, cf. http://chartsbin.com/view/7j7, ChartsBin statistics collector team 2010, Linguistic Diversity Index, ChartsBin.com, viewed 5th October, 2016. 
prend pas en compte la diversité interne des phylums du continent africain, ni leur profondeur historique.

Si les langues amérindiennes attestent autant de familles distinctes, c'est parce que toutes les tentatives de regroupement de niveau supérieur ont systématiquement été rejetées. Est-ce que cela signifie qu'à profondeur identique ${ }^{8}$ les langues africaines ont une diversité linguistique moindre ?

Il existe une technique développée dans le cadre des études typologiques pour calculer la diversité génétique en prenant en compte la complexité interne du groupe afin d'établir des échantillons des langues équilibrés. Rijkhoff et al. (1993 ; 1998) ont développé ce type de calcul pour ce qu'ils nomment des échantillons de variétés, i.e. des échantillons pour des études typologiques qui tendent à appréhender la diversité structurelle des langues. Ces calculs ont été développés pour éviter de biaiser les résultats typologiques. L'idée étant qu'avec des échantillons mal calibrés, la sur- ou sous- représentation d'un groupe de langues peut avoir des incidences sur le degré de fréquence d'une structure dans un échantillon. La diversité ou similarité structurelle interne d'une grande famille (profondeur) doit être prise en compte, autant que la diversité ou la similarité structurelle due à des apparentements distincts.

Notre propos ici n'est pas de savoir si cette méthode est meilleure qu'une autre pour les études typologiques, mais de regarder ce que ces calculs nous apprennent sur la diversité interne des regroupements linguistiques?.

Ce mode de calcul est utilisé pour définir le nombre de langues qu'il faut intégrer dans une analyse typologique de variété pour un même apparentement. Ce que nous interprétons comme : plus la structure interne de la famille est complexe, plus il est probable d'y trouver des différences structurelles. Indépendamment des critiques que l'on peut faire sur la classification des langues proposées par Ruhlen sur laquelle est basé le calcul présenté dans le tableau 1, on voit que le groupe de langues qui présente la plus grande diversité linguistique interne est la famille amérinde (178.44), les autres familles du continent présentent des degrés de diversité moindre (Eskimo-aleut 3.34 ; Na-dene 9.44). Pour le continent africain, aucun groupe n'a un degré de diversité très élevé, mais chacun a un degré relativement important par rapport à l'ensemble des groupes du tableau : Niger-Kordofanien 90.38 ; Afro-asiatique 55.53 ; Nilo-sahara 42.18, si l'on excepte le groupe khoisan (6.97). Ce dernier est maintenant, nous l'avons vu, divisé en trois familles distinctes

8 Ici le terme de profondeur renvoie soit à une notion temporelle, dans le cadre des arbres construits à partir de méthodes phylogénétiques où la longueur des branches correspond à un calibrage temporel ; soit, dans le cadre de méthodes de linguistique historique, à la complexité des regroupements basée sur des pourcentages de ressemblances, la profondeur se mesurant en nombre de nœuds entre la protolangue et les langues actuelles.

9 Une autre approche de ce type a été faite par Bell (1978). Il proposait de distinguer des groupes de façon arbitraire tous les 3500 ans à l'intérieur de chaque phylum. 
sans apparentement de niveau supérieur. Ainsi, si l'on croise les diversités interne (profondeur) et externe (nombre d'apparentements distincts), la diversité génétique reste importante sur le continent africain.

Tableau 1 - Taux de diversité linguistique interne calculée à partir des familles de langues établies par Ruhlen (1987), d'après Rijkhoff et Bakker (1998, p. 185).

\begin{tabular}{|l|c|}
\hline Phylum & $\begin{array}{c}\text { diversité } \\
\text { linguistique }\end{array}$ \\
Afro-asiatique & 55.53 \\
Altaïque & 14.79 \\
Amérinde & 178.44 \\
Australien & 67.58 \\
Austrique & 137.41 \\
Caucasien & 8.54 \\
Chukchi-Kamchatkan & 2.47 \\
Elamo-Dravidien & 7.43 \\
Eskimo-Aleut & 3.34 \\
Indo-Hittite & 39.71 \\
Indo-Pacifique & 124.79 \\
Khoisan & 6.97 \\
Sumérien & 0.00 \\
Ket & 0.00 \\
\hline
\end{tabular}

\begin{tabular}{|l|c|}
\hline Phylum & $\begin{array}{c}\text { diversité } \\
\text { linguistique }\end{array}$ \\
Nahali & 0.00 \\
Burrien & 0.00 \\
Méroitique & 0.00 \\
Basque & 0.00 \\
Etrusque & 0.00 \\
Gilyak & 0.00 \\
Na-dene & 0.00 \\
Niger-Kordofanien & 9.44 \\
Nilo-Saharien & 90.38 \\
Pidgins et Créoles & 42.18 \\
Sino-tibétain & 38.57 \\
Uralique-Yukaghir & 4.93 \\
\hline \multicolumn{2}{|c|}{ Total } \\
\hline
\end{tabular}

Pour les isolats, dans ce mode de calcul, même avec une diversité interne nulle (0.00), ils doivent faire partie intégrante des échantillons de langues des analyses typologiques. En effet, le calcul de la diversité interne des apparentements est utilisé pour ne pas sur- ou sous-représenter un groupe, mais chaque groupe doit avoir au moins un représentant dans l'échantillon. Donc idéalement tous les isolats doivent être intégrés dans les analyses typologiques de variétés.

Sur ce point, les langues parlées aux Amériques présentent effectivement la plus grande diversité, même sur la base de la classification de Ruhlen, synthétique et largement controversée. D'autres zones présentent également une diversité importante : l'Asie du Sud Est, l'Inde et l'océan pacifique, et l'Afrique qui ne peut être écartée.

Le troisième et dernier type de diversité linguistique que l'on peut distinguer est la diversité structurelle, diversité que tente d'appréhender la typologie. Dans 
les travaux sur les LED, nous avons vu qu'un parallèle est fait entre disparition de langues et perte de la diversité. Cependant, il n'est pas certain que la diversité structurelle doive nécessairement se trouver dans les LED.

Le basque à lui seul a permis, très tôt, la découverte de structure ergative pour les linguistes européens. Cette construction est restée typologiquement rare jusqu'à l'intégration d'un plus large éventail de langues ; avec notamment la description des langues australiennes et amérindiennes qui attestent pour beaucoup ce type de structure syntaxique. La multiplication des descriptions a donc permis de montrer d'une part que ce type de structure n'est pas marginal, mais également qu'il est complexe et diversement exprimé dans les langues.

Les langues « importantes » pour l'appréhension de la diversité structurelle ne sont pas non plus nécessairement des langues « exotiques ». L'utilisation du verbe faire comme auxiliaire dans les propositions interrogatives et négatives est un trait typologiquement rare attesté dans les langues papoues, mais également en anglais, qui n'est ni une langue en danger, ni une langue exotique. Wohlgemuth (2010) confirme en se focalisant sur les traits typologiques rares que la diversité structurelle n'est pas inhérente aux LED, ni aux langues à petites communautés.

The fact that one finds several large languages ranking high in the rarity index level list prohibits any generalization along the lines that rara would only or predominantly be found in small languages. (Wohlgemuth 2010, p. 265-6)

La description des LED est certes importante pour ne pas ignorer une diversité structurelle qui peut potentiellement être attestée uniquement dans ces langues. Pour autant, la description des LED peut aussi venir fausser les analyses typologiques, si leurs particularités ne sont pas prises en compte. Creissels, dans ce même numéro, montre que l'intégration des langues moribondes dans les analyses typologiques pose des problèmes à différents niveaux, et qu'elle peut être dommageable sur les généralisations qu'elles pourraient impliquer. Trudgill (2004, p. 318) indique que les LED ont généralement tendance à développer des systèmes phonologiques atypiques. Wohlgemuth (2010), Nettle (1999), Thurston (1987, 1992) montrent également que les petites communautés modifient leur langue d'une façon particulière, et ce que leur langue soit en danger ou non. Ces modifications sont plus rapides et se font selon des voies parfois inattendues typologiquement rares ou contraires aux corrélations déjà établies en typologie. Ces changements s'expliquent par la taille même de la communauté (Wohlgemuth 2010 ; Nettle 1999), mais aussi par des pratiques sociales propres à certaines de ces petites communautés, comme c'est le cas en Papouasie et Australie (Evans 2011 ; Thurston 1987, 1992).

En conséquence, si l'Afrique partage autant de diversités linguistiques que d'autres régions du monde dans lesquelles les LED sont prioritairement décrites, la 
différence notable que l'on peut mettre en avant est le plus grand nombre d'isolats recensé ailleurs qu'en Afrique. La description des LED dans le cadre de la préservation ou de la description d'une diversité linguistique menacée doit être envisagée avec nuance et ne pas laisser de côté des langues moins menacées à court terme qui sont aussi importantes, si ce n'est plus, pour une meilleure appréhension de la diversité linguistique, avec bien sûr une attention toute particulière aux isolats.

Dans la section suivante, nous revenons sur deux particularités des langues africaines qui doivent être prises en compte pour l'évolution de la situation des langues sur ce continent : les particularités du multilinguisme africain et les changements sociaux en cours qui ont des incidences sur la dynamique des langues.

\section{LES LANGUES AFRICAINES EN PLEINE VITALITÉ,} DANS UNE DYNAMIQUE SANS CONCURRENCE?

\subsection{Des critères linguistiques à reconsidérer}

Comme le notent Connell (2007) et Lüpke (2015), la situation du multilinguisme en Afrique a des incidences très importantes sur la vitalité des langues, mais ces situations sont souvent mal connues et peu documentées.

A second, and more important, reason may be the relatively high degree of multilingualism found in many parts of Africa, which may tend to obscure the degree of language shift in progress. However perhaps most important, [...], to a large extent this perception may be no more than a reflection of our lack of knowledge of the linguistic situation in a great many parts of Africa; a lack of available information. (Connell 2007, p. 163)

Finally, patterns of multilingualism may change rapidly, e.g. in northern Nigeria, where numerous Chadic and Benue-Congo languages may vanish in the near future. (Dimmendaal et Voeltz 2007, p. 606)

Généralement, les études faites sur cette question sont menées sur l'ensemble du pays ou dans une zone géographique précise. À la différence des enquêtes rassemblée par Calvet et Moussirou-Mouyama (2000) ou celles de Dreyfus et Juillard (2005), les enquêtes sur le multilinguisme sont rarement basées sur une observation des pratiques, et toutes reposent sur une représentation hiérarchisée des langues ${ }^{10}$.

10 Mon propos ici n'est pas de nier les enjeux de pouvoir sous-jacents au choix des langues selon les contextes, mais d'observer les pratiques des locuteurs en prenant en compte le répertoire linguistique de chacun sans chercher à hiérarchiser les langues en pratique, d'observer les différences de choix et de les comprendre. 
[...] cette diversification de la nomenclature en types de $«$ langues $»^{11}$ doit plus à l'exploitation des questionnaires d'enquête et à la projection cartographique du traitement des réponses qu'à une réelle observation des pratiques. (Renaud 1998, p. 20)

Les enquêtes concernant le répertoire des locuteurs sont encore plus rares (Canut 1996). Ces observations du répertoire linguistique sont essentiellement basées sur des analyses sociolinguistiques. Elles tentent d'appréhender les usages réels faits de cette multitude de langues et de distinguer les situations dans lesquelles une langue est utilisée.

Les conséquences que l'utilisation d'une langue - dans des situations particulières et souvent très spécifiques - a sur la structure de cette langue ne sont jamais prises en compte. Pourtant, à l'intérieur du répertoire linguistique, si plusieurs langues sont plus largement utilisées, d'autres sont cloisonnées à des situations de communication très restreintes. La multiplication de langues à large éventail d'utilisation peut impliquer la réduction du champ d'action de langue(s) du répertoire ou la suppression d'autres langue(s) déjà restreinte(s) à des situations spécifiques. Par conséquent, la situation des langues africaines implique d'une part la prise en compte des répertoires linguistiques dans la problématique des LED. Elle doit, d'autre part, poser la question de la structure qu'attestent réellement les langues restreintes à des situations d'échanges très spécialisés.

Sur ce point, les observations que j'ai pu effectuer sur le terrain lors d'enquêtes en vue de la description du kobiana sont intéressantes. Le kobiana est une langue atlantique (groupe nyun-buy) pratiquée depuis longtemps par une petite communauté en bordure du fleuve Cacheu en Guinée Bissau. Les Kobiana, majoritairement animistes, sont depuis leur installation dans cette région considérés par d'autres populations comme détenant la corne ${ }^{12}$ ayant la plus grande puissance. Des pèlerinages réguliers impliquant des populations (Baïnouk, Diola feloup, et Manjak, au moins) provenant du Sénégal comme de la Guinée Bissau sont organisés en pays Kabuy pour réaliser des offrandes auprès de leur autel Mama Jombo. La force de leur puissance est ainsi reconnue bien au-delà du petit groupe que constituent les Kobiana eux-mêmes, et cette reconnaissance va au-delà de la région et du pays ${ }^{13}$.

11 Ici l'auteur renvoie aux termes de langues maternelles, vernaculaires, véhiculaires, nationales, officielles...

12 Les cornes d'animaux constituent des réceptacles dans lesquels les puissances du culte pratiqué par les Kobiana sont installées. Elles sont utilisées comme autel pour les libations et les sacrifices.

13 Les relations entre ces différents groupes sont complexes, mais seuls les Baïnouk (Nyun) partagent avec les Kobiana des liens linguistiques étroits (même groupe) et une origine commune (traditions orales). Pour autant, les Baïnouk n'ont pas quitté le Sénégal. Les Felup et les Manjak parlent également des langues atlantiques, mais dans des groupes différents et distants de celui du kobiana. Si les Manjak vivent en Guinée Bissau, les Felup habitent des deux côtés de la frontière entre le Sénégal et la Guinée et résident dans des villages côtiers. 
La vitalité de ces pratiques laisse supposer que le kobiana, langue d'initiation et de communication avec la puissance incarnée dans la corne, survivra au moins dans ce registre dans la région pour les offrandes et les sacrifices. Pour autant, elle ne sera très certainement plus utilisée dans les échanges entre Kobiana dans les villages (Guinée Bissau), où elle est déjà en concurrence avec la langue véhiculaire du pays, le créole bissau-guinéen.

L'observation des répertoires linguistiques dans les zones rurales et dans les villes ne conduira pas nécessairement au même constat. Cette observation ne doit pas conserver une approche hiérarchisée des langues, telle qu'elle est le plus souvent projetée sur l'Afrique, i.e. une langue officielle haute mais peu maîtrisée par la plupart des locuteurs, une langue véhiculaire et une langue maternelle bien maîtrisées.

Les Kobiana qui vivent dans les centres urbains sénégalais, ont une langue maternelle revendiquée comme étant le kobiana, mais ils ne la maitrisent pas pleinement, dans le sens où ils ne l'utilisent que dans certains registres, et changent de langue selon l'objet de l'interaction. Les variétés urbaines, créoles, langues véhiculaires (diola et wolof pour les habitants de la Casamance $^{14}$ ), i.e. les langues pratiquées le plus régulièrement, le plus quotidiennement sont les langues qu'ils utilisent dans un plus large registre, alors qu'ils se considèrent comme de « mauvais locuteurs » de ces langues ou du moins de certaines d'entre elles. Ils disent souvent ne pas toutes les maitriser correctement. Les Kobiana, par exemple pratiquent toutes générations confondues le créole, qu'ils soient en Guinée Bissau ou au Sénégal. Ils se considèrent, généralement, comme bons locuteurs de cette langue. En ce qui concerne le wolof, pour les Kobiana du Sénégal, les plus âgés ne le pratiquent pas ou peu, mais les autres générations l'utilisent régulièrement. Le plus souvent ces derniers se considèrent pourtant comme de mauvais locuteurs de wolof, parce qu'ils disent « le mélanger avec d'autres langues ». Ce discours sur le wolof urbain, présenté comme étant un « mauvais » wolof, est tenu par nombre de Sénégalais, y compris des Dakarois d'origine wolof. Ce discours est essentiellement basé sur une comparaison avec « le wolof rural » (toutes variétés rurales confondues) posé comme langue de référence, désigné comme étant un wolof « pur », puisque moins imprégné d'emprunts à d'autres langues, comme le français ou l'anglais entre autres.

L'observation de l'utilisation des langues du répertoire linguistique devrait apporter un nouveau regard sur la situation des LED en Afrique et permettre de repérer les langues pour lesquelles on peut considérer qu'il ne reste finalement

14 Pour compléter le répertoire linguistique de ces locuteurs, pour tous il faut au moins ajouter le manjak, langue de quartier dans les zones urbaines et langue de la communauté environnante en pays Kabuy (Guinée Bissau). Ensuite, pour chaque locuteur d'autres langues peuvent être ajoutées. 
plus de locuteurs traditionnels (ou fluent speaker) ou très peu et pour lesquelles on ne trouve finalement que des semi-locuteurs, c'est-à-dire des locuteurs qui ne peuvent utiliser la langue que dans des situations particulières et ont perdu les compétences ou n'ont jamais appris les structures de cette langue pour l'utiliser dans d'autres contextes ${ }^{15}$. Cette situation vaut bien sûr pour les langues menacées à court terme, mais pourrait être étendue à des langues considérées pour l'instant comme menacées à plus ou moins moyen terme.

\subsection{Des critères sociaux à prendre en compte}

Un autre facteur doit également être pris en compte si l'on veut véritablement observer la situation des LED en Afrique avec des critères mieux adaptés.

Nous avons vu que dans les centres urbains, les langues les plus répandues, les langues qui se véhicularisent, sont des langues africaines, ce qui les met en concurrence avec d'autres langues africaines. Or, il semblerait que cette situation soit en train d'évoluer. L'Afrique sub-saharienne connaît depuis 1990 une très forte accélération dans deux domaines. Ces deux domaines sont liés à la croissance économique inégale, mais générale au continent, qui conduit d'une part à l'émergence d'une classe moyenne et, d'autre part à une augmentation du taux d'urbanisation encore jamais connue pour ce continent.

L'observation des répertoires linguistiques doit par conséquent être différente dans les zones rurales et les zones urbaines. Les langues du répertoire ne sont nécessairement pas les mêmes dans ces zones, et certaines langues tendent à avoir des registres d'utilisation de plus en plus étendus selon les zones.

\section{Émergence d'une classe moyenne en Afrique}

Entre 1990 et aujourd'hui, la classe moyenne en Afrique a doublé. On considère aujourd'hui qu'un tiers de la population africaine appartient à cette classe. Les définitions du terme de « classe moyenne » en Afrique sont assez différentes selon les sources. Les divergences se cristallisent sur le revenu minimum au-delà duquel le foyer est considéré comme ayant dépassé le seuil de pauvreté. Dominique Darbon et Joan Bardeletti le définissent à la suite de leur projet sur les classes moyennes en Afrique comme toutes «personnes disposant d'une somme d'argent qu'elles peuvent choisir de dépenser ou non une fois qu'elles ont satisfait à leurs besoins essentiels $»^{16}$. Sur cette base, ils proposent différentes sous-catégories.

Sur le plan linguistique, la principale modification qu' apporte l'émergence d'une classe moyenne est un accès plus large des jeunes générations à la scolarisation.

15 La désignation des types de locuteurs repose sur la typologie dressée par Bert et Grinevald (2012).

16 Cf. http://www.classesmoyennes-afrique.org/fr 
Certes, la durée de cet accès reste différente selon les sous-catégories de classe moyenne, notamment pour la «catégorie flottante ${ }^{17}$. Pour autant, depuis le début des années 2000, la société africaine change de visage. L'accès à la scolarisation et l'allongement de la durée de scolarisation d'une plus large part de la population accroissent l'accès à la langue officielle.

Il faut savoir que l'Afrique sub-saharienne reste encore une des régions du monde dans laquelle peu d'enfants sont scolarisés dans l'enseignement primaire. Dans la section précédente, nous avons considéré différents facteurs qui permettaient d'expliquer la vitalité de certaines langues locales face aux langues officielles, comme une colonisation d'exploitation ou l'ancienneté des langues véhiculaires africaines. Mais il ne faut pas non plus négliger le faible degré de scolarisation. Si peu de personnes pratiquent les langues officielles, c'est également parce qu'une infime part de la population avait jusqu'à présent accès à ces langues via la scolarisation. La scolarisation d'un plus grand nombre d'enfants et une durée des études plus longue auront et ont déjà dans certains pays africains des incidences sur la dynamique entre les langues locales et la langue officielle. Si le phénomène est récent en Afrique (2000), il progresse de façon exponentielle et doit nécessairement être pris en compte.

\section{Une urbanisation galopante}

Autre « retard » que 1'Afrique est en train de rattraper, 1'urbanisation. Il y a 50 ans, moins de $15 \%$ de la population habitait en zone urbaine, ils sont aujourd'hui environ $40 \%$ ( \pm 450 millions) et les prévisions indiquent qu'il y en aura 3 fois plus en $2050^{18}$.

La configuration des villes change également. En 1950, aucune ville n'avait plus d'un million d'habitants. En 2015, il y a environ 50 villes qui ont plus d'un million d'habitants. Il existe désormais des mégalopoles, mais aussi tout un réseau de petites villes, ce qui n'était pas le cas jusqu'à il y a peu, où sorti des capitales administratives, économiques et politiques qui peuvent dans certains pays être situées dans des villes différentes, on ne trouvait pas de villes, mais de grands bourgs. Sur la carte ci-dessous, on peut également voir que les villes qui ont doublé leur population en l'espace de 10 ans sont pour la plupart situées en Afrique sub-saharienne.

$17 \mathrm{La}$ « catégorie flottante » est la frange de la classe moyenne qui peut très vite retomber dans la pauvreté du fait d'une situation plus instable (emploi, situation familiale, lieu de vie...).

18 En 2050, la croissance de la population africaine actuelle permet d'envisager une population de 2,4 milliards dont $60 \%$ seront dans des zones urbaines. Cf. entre autres http://esa.un.org/ unpd/wup/. 


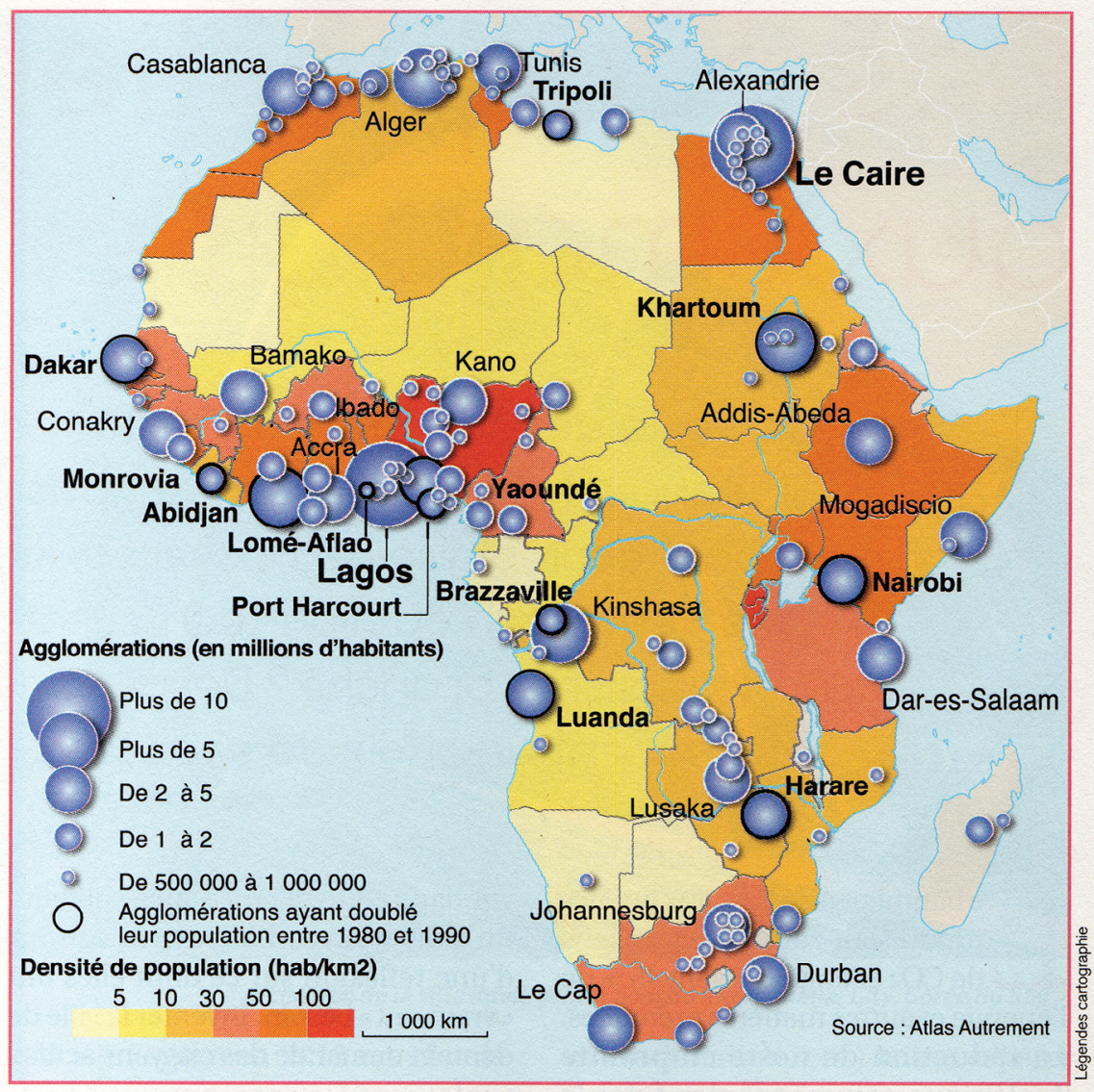

Carte 1 - Les grandes villes d'Afrique (Atlas autrement, 2015)

La conjugaison de l'émergence d'une classe moyenne et d'une forte urbanisation redessine le paysage même des villes. Les quartiers ne sont plus des rassemblements de personnes issues d'une même région ou d'un même village. Certains quartiers de la ville africaine reflètent désormais différents niveaux sociaux, et ressemble plus aux autres villes du monde, dans lesquelles il existe des quartiers aisés, des quartiers de classes moyennes et des zones périurbaines dans lesquelles se rassemble la population la plus pauvre.

La motivation de l'exode rural change également. Les jeunes continuent à se rendre en ville pour trouver du travail, mais également pour avoir accès « à la modernité », pour fuir le poids des traditions et tendent, lorsqu'ils s'installent en ville, à se regrouper en classes d'âge, mais plus nécessairement avec les gens de leur village (ou région). 
Ces changements sont assez récents et ont été extrêmement rapides. Les impacts qu'ils ont et auront sur la dynamique des langues et sur le répertoire linguistique ne sont pas encore bien établis ni décrits, mais il est indéniable qu'ils vont changer les équilibres décrits jusqu'à présent.

\section{Conclusion}

Dans cet article nous avons voulu montrer que la position de l'Afrique dans la problématique des LED, bien que différente d'autres régions du monde, ne doit pas pour autant être considérée comme moins préoccupante. Les changements en cours modifient la situation des langues du continent, y compris celles que l'on pensait moins menacées jusqu'à présent.

Le nouveau regard que l'on peut porter sur cette situation s'explique par un changement de perspective dans les analyses sur les langues africaines. La révision des classifications des langues basées sur l'intégration des dernières avancées montre que la tendance dans les questions de filiations des langues africaines est à l'éclatement des phylums établis jusqu'à présent par Greenberg. L'urbanisation et l'émergence d'une classe moyenne récentes et les conséquences sur les équilibres établis jusque-là doivent absolument être pris en compte.

Cette reconsidération de la position de l'Afrique ne tient pas qu'à des changements. Les critères utilisés pour considérer la position de l'Afrique dans cette problématique ne sont pas nécessairement les plus adaptés. En Afrique, les langues ne disparaissaient pas parce qu'elles sont en concurrence avec les langues des anciens colonisateurs. Cette situation reste vraie dans certains pays encore faiblement urbanisés et dans les zones rurales. En revanche, cette expansion de langues locales met en concurrence des langues africaines face à d'autres langues africaines. Elle fragilise alors directement d'autres langues africaines qui ne sont pas nécessairement des langues apparentées aux langues véhiculaires, ni des langues qui leur sont structurellement identiques. Les situations de multilinguisme spécifiques au continent africain doivent alors être prises en compte, afin d'adapter les critères de vitalité des langues à ces situations.

Ce nouveau regard sur les LED en Afrique n'a pas pour objectif de lancer des plans de revitalisation tous azimuts, mais bien de rendre leur place aux langues de ce continent dans la prise en compte de la diversité linguistique et de sa documentation. Ma position face à la sauvegarde des langues est la même que celle exprimée par Calvet (2002, p. 96) « Les langues sont faites pour servir les hommes, et non pas l'inverse. Cela signifie tout simplement qu'une langue n'a pas à être protégée par principe mais qu'elle doit l'être chaque fois que ses locuteurs en ont besoin. » Sur ce point, il y a en Afrique peu de communautés qui expriment ce besoin. 
Mais la description des LED n'implique pas nécessairement leur documentation, ni leur revitalisation. Par ailleurs, les questions adressées dans cet article montrent que l'intégration des LED soulève des questions et offre des angles d'observation auxquels la description de langues non menacées ne conduit pas nécessairement.

\section{RÉFÉRENCES}

Batibo, Herman, 2005. Language decline and death in Africa: causes, consequences, and challenges, Clevedon, Buffalo, Toronto, Multilingual Matters LTD.

Bell, Alan, 1978. « Language Samples », J. Greenberg et al. (eds.), Universals of human languages: method - theory, Stanford, Stanford University Press, 1, 123-56.

Bert, Michel \& Colette Grinevald, 2012. "Proposition de typologie des locuteurs de LED », Faits de langues 35/36, 117-132.

Blench, Roger, 2007. "Endangered languages in West Africa », M. Brenzinger (ed.) Language diversity endangered, La Hague, Mouton de Gruyter, 140-162.

-, 2013. "Why is Africa so linguistically undiverse? Exploring substrates and isolates », Journal of the Association for the Study of Language in Prehistory, XVIII (50th Anniversary of J. H. Greenberg's The Languages of Africa (1963)), 43-78.

Calvet, Louis-Jean \& Auguste Moussirou-Mouyama, 2000. Le plurilinguisme urbain. Québec, Didier Erudition.

Canut, Cécile, 1996. Dynamiques linguistiques au Mali. Langues et développement. Paris, Didier Érudition.

Connell, Bruce, 2007. « Endangered Languages in Central Africa », M. Brenzinger (ed.) Language diversity endangered, La Hague, Mouton de Gruyter, 163-78.

Crystal, David. 2000. Language death, Cambridge, Cambridge University Press.

Dimmendaal, Gerrit J., 2008. « Language Ecology and Linguistic Diversity on the African Continent. » Language and Linguistics Compass 2 (5), 840-858.

Dimmendaal, Gerrit J. \& Erhard Friedrich Karl Voeltz, 2007. " Africa », Christopher Moseley (ed.) Encyclopedia of the World's Endangered Languages, Oxon, Routledge, 579-634.

Dreyfus, Martine \& Caroline Juillard, 2005. Le plurilinguisme au Sénégal : langues et identités en devenir, Paris, Karthala.

Ehret, Christopher, 2000. «Langage and history », B. Heine \& D. Nurse, African Languages: An Introduction, Cambridge, Cambridge University Press, 272-297.

Evans, Nicholas, 2011. Dying Words: Endangered Languages and What They Have to Tell Us, John Wiley \& Sons.

Ferro, Marc, 1994. Histoire des colonisations : des conquêtes aux indépendances $X I{ }^{e}-X X^{e}$, Paris, Le Seuil.

- 2003. Le livre noir du colonialisme. XVI $-X X I^{e}$ siècle : de l'extermination à la repentance, Paris, Robert Laffont.

Greenberg, Joseph H, 1956. « The Measurement of Linguistic Diversity », Language 32 (1), 109-115.

- 1963. The languages of Africa, Vol. 1, Part II, International Journal of American Linguistics 29.

Heine, Bernd \& Derek Nurse, 2000. African Languages: An Introduction, Cambridge, Cambridge University Press.

Kaufman, Terrence, 1990. « Language history in South America: What we know and how to know more ", Dorian L. Payne (ed.), Amazonian linguistics: Studies in lowland South American languages, Austin, University of Texas Press, 13-74

- 1994. « The native languages of South America.» C. Mosley \& R. E. Asher, Atlas of the world's languages, London, Routledge, 46-76. 
Kießling, Roland, Maarten Mous, \& Derek Nurse, 2008. « The Tanzanian Rift valley area », B. Heine \& D. Nurse (eds), A linguistic Geography of Africa, Cambridge, Cambridge University Press, 186-227.

Krauss, Michael E., 1992. «The World's Languages in Crisis », Language 68 (1), 4-10.

Lüpke, Friederike, 2015. " Ideologies and Typologies of Language Endangerment in Africa » J. Essegbey, B. Henderson \& F. Mc Laughlin (eds), Language Documentation and Endangerment in Africa, Culture and Language Use 17, John Benjamins Publishing Company, 59-106.

M'Bokolo, Elikia, 1995. Afrique Noire. Histoire \& Civilisation jusqu'au XVIII siècle. Vol. 1. Paris, Hatier.

Moore, Joslin L., Lisa Manne, Thomas Brooks, Neil D. Burgess, Robert Davies, Carsten Rahbek, Paul Williams, \& Andrew Balmford, 2002. "The Distribution of Cultural and Biological Diversity in Africa ", Proceedings of the Royal Society of London B: Biological Sciences 269 (1501), 1645-153.

Mous, Maarten, 2003. "Loss of linguistic diverstiy in Africa », M. Janse \& T. Sijmen (eds), Language death and language maintenance: Theorical, pratical and descriptive approaches, Amsterdam, John Benjamins Publishing, 157-170.

Mufwene, Salikoko S., 2003. The Ecology of Language Evolution, Cambridge, Cambridge University Press [First published 2001].

Nettle, Daniel, 1999. Linguistic Diversity, Oxford, Oxford University Press.

Nettle, Daniel \& Suzanne Romaine, 2000. Vanishing Voices : The Extinction of the World's Languages, Oxford, Oxford University Press.

Nichols, Johanna, 1999. Linguistic Diversity in Space and Time, Chicago, University of Chicago Press.

Renaud, Patrick, 1998. «L'invention du verbe : d'une linguistique en Afrique à une linguistique de l'Afrique ", Faits de langues 6 (11), 13-46.

Rijkhoff, Jan \& Dik Bakker, 1998. " Language sampling », Linguistic Typology 2 (3), 263-314.

Rijkhoff, Jan, Dik Bakker, Kees Hengeveld \& Peter Kahrel, 1993. «A Method of Language Sampling ", Studies in Language 17 (1), 169-203.

Sands, Bonny, 2009. «Africa's Linguistic Diversity », Language and Linguistics Compass 3 (2), 559-580.

Thurston, William R., 1987. Processes of change in the Languages of North-western New Britain, Canberra, Pacific Linguistics.

- 1992. "Sociolinguistic typology and others factors effecting change in north-western New Brintain, Papa New Guinea », T. E. Dutton (ed), Culture change, Language change. Case Studies from Melanesia, Canberra, Pacific Linguistics, 123-139.

Vigouroux, Cécile \& Salikoko S. Mufwene, 2008. "Colonization, Globalization and Language Vitality in Africa: An Introduction », C. Vigouroux \& S. S. Mufwene (eds), Globalization and Language Vitality: Perspectives from Africa, London, Continuum, 1-31.

Wohlgemuth, Jan, 2010. « Language Endangerment, Community Size and Typological Rarity ", J. Wohlgemuth \& M. Cysouw (eds), Rethinking Universals: How Rarities Affect Linguistic Theory, Empirical Approaches to Language Typology 46, Berlin, New York, Walter de Gruyter, 255-277. 